\title{
COSMOLOGY WITH NEARBY DWARF GALAXIES: THE VIEW FROM HST
}

\author{
ELINE TOLSTOY \\ Space Telescope - European Coordinating Facility \\ Karl-Schwarzschild str. 2 \\ D-85748 Garching bei München, Germany
}

\section{Colour-Magnitude Diagrams: What they tell us.}

Stellar Evolution theory is based upon well understood physics and provides clear predictions as to how a Colour-Magnitude Diagram (CMD) will change due to effects of age and metallicity. The theory has been tested by looking at nearby coeval star clusters. The power of applying CMD analysis to galaxies has been demonstrated in studies of the Carina dSph (Smecker-Hane et al. 1996). In Carina the observation of separate, distinct Main Sequence (MS) Turnoffs has forced us to believe that this small, nearby companion of our Galaxy has had a very complex star formation history. No similar direct evidence for "bursting" behaviour on a global scale has been seen indisputably in larger systems. Partly this is due to the greater distances of larger systems, but also to the complications in distinguishing old star formation events ( $>1$ Gyr old) in systems which are currently forming stars. Where we lack MS turnoffs we have to resort to statistical modeling of the CMD. This has been applied using a a number of different approaches, but all generally based on Monte-Carlo techniques (e.g. Tosi et al. 1992; Bertelli et al. 1992; Tolstoy \& Saha 1996). However, even using these more sophisticated analysis techniques, it is difficult to find unique solutions. This is mostly due to the age-metallicity degeneracy on the Red Giant Branch (RGB). The RGB is usually the most populated, easiest to observe phase of stellar evolution. The Carina CMD reveals the dangers of blindly interpreting the RGB, because from the RGB alone it is impossible to extract the information revealed by the MS Turnoffs.

\section{Recent Results from the Hubble Space Telescope}

The HST is an invaluable resource for understanding the evolution of the resolved stellar populations in nearby galaxies. Crowding problems plague ground-based studies of all but the closest, least densely populated systems. The spatial resolution of HST $\left(0.1^{\prime \prime}\right)$ minimises image crowding and makes it possible to detect fainter and thus older stars, and look farther back into the past star formation history of a galaxy. It has been postulated that nearby dwarf irregular galaxies are the most likely candidates for the remanents of the high redshift faint blue galaxy population (Koo, Guzman, this volume). The only way to test this unambiguously is to make deep CMDs, detecting more than just the RGB.

Recent HST CMDs are starting to provide a more detailed understanding of galaxy evolution (e.g. Armandroff, Grebel, this volume). The more carefully we look at nearby star-forming galaxies, the more detail we find. A few recent examples of HST/WFPC2 results are:

Sextans A (Dohm-Palmer at al. 1997): This appears to be a typical low metallicity $(Z=0.001)$ star forming galaxy. It's WFPC2 CMD shows a galaxy that has most recently (over the last Gyr) been actively forming stars with a rate that has fluctuated with time by a factor of $2-3$ across the galaxy. This is one of the first CMDs to definitively show the gap between the MS and the Blue Loop (BL) Helium Burning stars enabling a more accurate disentangling of the recent star formation history. This is a testament to the superior resolving powers of HST.

Pegasus dI (Gallagher et al. 1997, submitted): From the WFPC2 CMD of this galaxy we see it has a similar metallicity to Sextans A, but a much lower current rate of star formation. From the well defined RGB, and AGB as well as Red Clump stars it is clear that this galaxy has 
been forming stars further back in time than the observed MS, but the age range and intensity of the star formation cannot be disentangled without a reliable model for the chemical evolution versus time of this galaxy. If a rapid early chemical enrichment is assumed, with very little further self-enrichment, then this can be only a 3-4 Gyr old galaxy. However if we assume a more gradual enrichment, the galaxy can contain much older stars.

Leo A (Tolstoy et al. 1997, in preparation): The WFPC2 CMD shows that this galaxy has a low current star formation rate, although not as low as Pegasus, and an extremely low metallicity $(\mathrm{Z}=0.0004)$. As in Sextans $\mathrm{A}$, there is a well defined BL sequence, well separated from the Main Sequence. We also see what appears to be a Red Clump. What is remarkable about this CMD is the extremely blue, and narrow RGB. It is necessary to go to the lowest metallicity stellar evolution tracks available to model this, making it impossible to quantify the possible effects of chemical evolution. The red limit of the RGB, gives an age limit for the oldest stars of about a few Gyr. Another result from this new CMD, and the detection of a Red Clump, is that the Cepheid distance to Leo A (Hoessel et al. 1994) is clearly wrong, by about 2.5 magnitudes. The reasons for this are unclear.

VII Zw403 (Lynds et al. 1997, in preparation): This is the nearest example of a Blue Compact Dwarf (BCD) Galaxy. The comparison between the WFPC2 CMDs of VII Zw403 and Sextans A is very revealing. The young, high mass stellar populations look very similar, including the differentiation between MS and BL. However the large difference in ratio of young stars in the $\mathrm{MS}+\mathrm{BL}$ and those in the RGB+AGB between these systems shows that these galaxies have had very different star formation histories in the recent past. In addition to a highly populated RGB VII Zw403 also has a strong AGB sequence. The uncertainties in modeling the AGB leads to a reluctance to rely on its interpretation, except to note that since the AGB is a very short-lived phase, to see so many is a strong indication that a "burst" of star formation has occured in the recent past (1-5Gyr ago). Modeling of the RGB supports (although not unambiguously) the idea that a burst of star formation occured within the last 2 Gyr (Hunter et al. 1996). Interestingly VII Zw403's CMD looks very similar to the CMD of the nearby dwarf irregular galaxy, NGC 6822 (Gallart et al. 1994), which supports the idea that BCDs and low surface brightness dIs are the same objects (Bergvall, this volume).

\section{The Big Picture}

To fit these results into a scenario which will provide an overall understanding of dwarf galaxy evolution Ferrara \& Tolstoy (1997, in preparation) have created a model which uses supernovae and stellar winds to create significant turbulent pressure in the ISM of a galaxy which effectively regulates star formation efficiency. The result of this is that the total mass of a galaxy determines how many stars a galaxy will form over time. The more massive a galaxy (total mass, dark plus visible) the more easily HI gas can stay concentrated in the centre, and thus star formation is more efficient, and the metallicity of the galaxy is enhanced. Thus the scatter in metallicity between galaxies of the same HI gas mass can be accounted for by variations in the dark matter content. From this model the observed global properties of mass and metallicity of dwarf galaxies seen today can be accounted for (e.g. van Zee this volume). It was also found, and subsequent hydro-simulations confirm (Ferrara \& MacLow 1997, in preparation), that it is very difficult to lose gas from a galaxy through supernovae explosions. Thus the total mass of a galaxy does not only regulate the mass loss from the galaxy, but also the overall star formation efficiency within the galaxy.

\section{References}

Bertelli G., Mateo M., Chiosi C., \& Bressan A. (1992) ApJ, 388, 400

Dohm-Palmer R., Skillman E., Saha A., Tolstoy E., Mateo M., Gallagher J., Hoessel J., Chiosi C. (1997) AJ, in press

Gallart C., Aparicio A., Chiosi C., Bertelli G., Vilchez J. M. (1994) ApJ, 425, 9

Hoessel J. G., Saha A., Krist J, Danielson G. E. (1994) AJ, 108, 645

Hunter D. A., Lynds R., Tolstoy E., O'Neil E. (1996) BAAS, 189, 3802

Smecker-Hane T.A., et al. (1996) From Stars to Galaxies, eds. Leitherer et al., p.328

Tolstoy E. \& Saha A. (1996) ApJ, 462, 672

Tosi M., Greggio L., Marconi G., Focardi P. (1991) AJ, 102, 951 

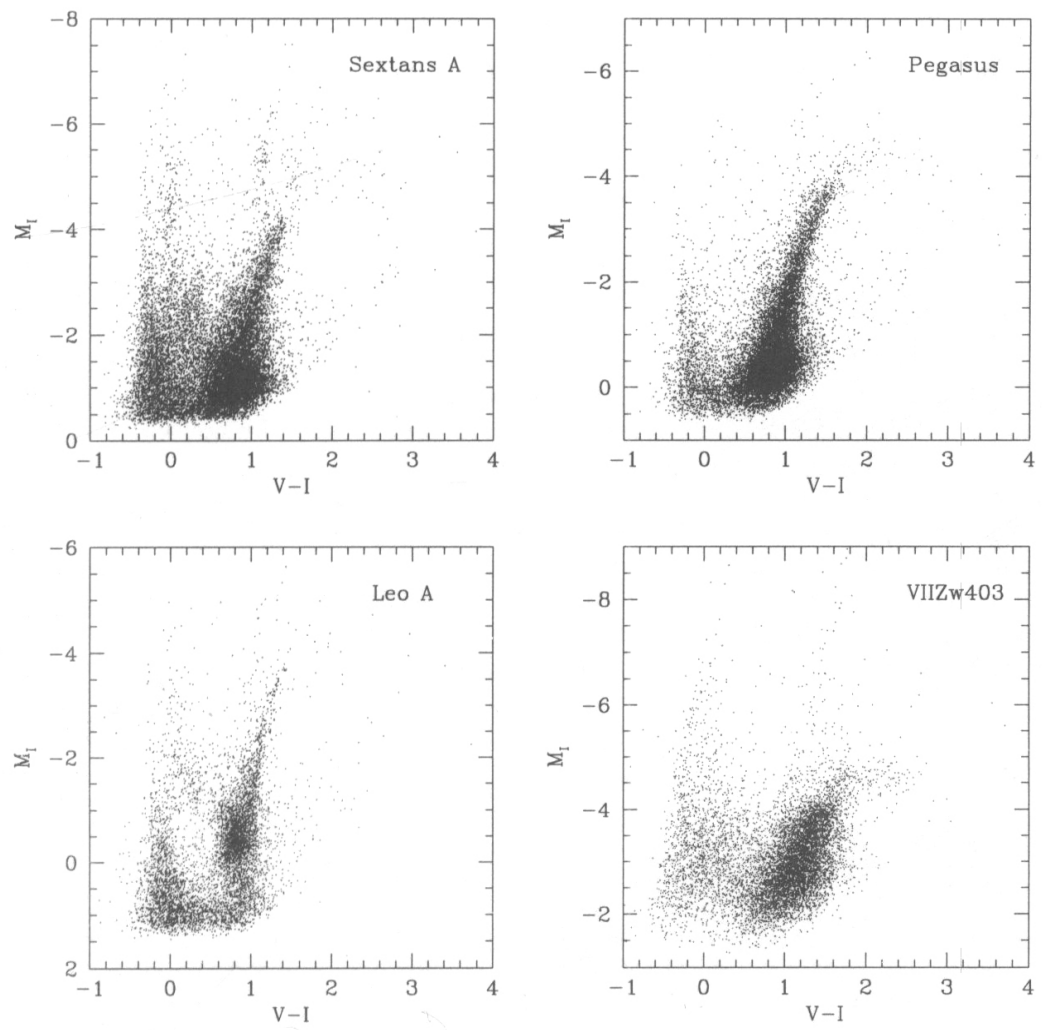

Fig. 1: HST-WFPC2 Colour-Magnitude Diagrams for four nearby star forming dwarf galaxies, as described in the text. The Red Clump is seen at $M_{I} \sim-0.5$, and the tip of the RGB is $M_{I} \sim-4$. The bifurcation of the "blue plume" into Main Sequence and the Blue Loop is most clearly seen in the Sextans A CMD. 